\title{
IMBEDDING FREE CYCLIC GROUP ACTIONS IN CIRCLE GROUP ACTIONS
}

\author{
JEFFREY L. TOLLEFSON
}

\begin{abstract}
Suppose a closed, orientable, irreducible 3-manifold $M$ admits a free cyclic group action of prime order. We consider the problem of determining when $M$ admits an effective action of the circle group $S O(2)$ in which the cyclic action is imbedded. The main result is that if the $Z_{k}$ action is " $Z$-classified", then it is weakly equivalent to a $Z_{k}$ action imbedded in an effective action of $S O(2)$ if and only if some homeomorphism generating the first $Z_{k}$ action is homotopic to the identity homeomorphism on $M$.
\end{abstract}

Let $Z_{k}$ be a cyclic group of prime order $k \geqq 2 . A Z_{k}$ action on a 3manifold $M$ is said to be proper if some homeomorphism generating the action is homotopic to the identity on $M$. Let $M^{*}$ denote the orbit space $M / Z_{k}$ and $\pi: M \rightarrow M^{*}$ denote the projection. We shall say that a $Z_{k}$ action on $M$ is $Z$-classified if there exists a commutative diagram

$$
\begin{aligned}
& M \rightarrow S^{1} \\
& \downarrow \pi \quad \downarrow p \\
& M^{*} \rightarrow S^{1}
\end{aligned}
$$

where $p: S^{1} \rightarrow S^{1}$ is the usual $k$-sheeted covering of the circle. In particular, this is always the case when $H_{1}\left(M^{*} ; Z\right)$ has no $k$-torsion [5].

$A Z_{k}$ action on $M$ is determined by a map $\mu: Z_{k} \times M \rightarrow M$, where $\mu(g)(x)=\mu(g, x)$ is a homeomorphism for each $g \in Z_{k}$ and $\mu\left(g \circ g^{\prime}\right)$ $=\mu(g) \circ \mu\left(g^{\prime}\right)$. The $Z_{k}$ actions determined by the maps $\mu, \nu: Z_{k} \times M$ $\rightarrow M$ are said to be weakly equivalent if there is a group automorphism $A: Z_{k} \rightarrow Z_{k}$ and a homeomorphism $H: M \rightarrow M$ such that $\mu(g)$ $=H^{-1} \circ \nu(A(g)) \circ H$ for all $g \in Z_{k}$.

THEOREM. Let $M$ be a closed, orientable, irreducible 3-manifold. A $Z$-classified free $Z_{k}$ action on $M(k \geqq 2$ and prime) is proper if and only if it is weakly equivalent to some $Z_{k}$ action embedded in an effective $S O(2)$ action on $M$.

Proof. Let $p: S^{1} \rightarrow S_{0}^{1}$ be the standard $k$-fold covering of the circle

Received by the editors March 13, 1970.

AMS 1969 subject classifications. Primary 5701, 5747; Secondary 5478, 5480, 5560. tions.

Key words and phrases. Finite cyclic group actions, 3-manifolds, circle group ac- 
$S_{0}^{1}$. There are maps $f$ and $\bar{f}$ such that the diagram

$$
\begin{aligned}
& M \stackrel{\bar{f}}{\rightarrow} S^{1} \\
& \downarrow \pi \underset{\downarrow}{\downarrow} p \\
& M^{*} \stackrel{f}{\rightarrow} S_{0}^{1}
\end{aligned}
$$

commutes. By the homotopy covering property, for any map $M^{*} \rightarrow S_{0}^{1}$ homotopic to $f$ (with the appropriate map $M \rightarrow S^{1}$ ) the diagram also commutes. Hence for some $a \in S_{0}^{1}$ we may assume that $F=f^{-1}(a)$ is a closed, connected, orientable, polyhedral, two-sided 2-manifold such that the homomorphism induced by inclusion $i_{*}: \pi_{1}(F) \rightarrow \pi_{1}\left(M^{*}\right)$ is one-to-one [7, Lemma 1].

Let $p^{-1}(a)=\left\{a_{i}\right\}_{i=1}^{k} \subset S^{1}$. By a suitable choice of labeling, we may let $A$ be the arc in $S^{1}$ with endpoints $a_{1}$ and $a_{2}$ such that no other $a_{i}$ lies on $A$. Let $U=\bar{f}^{-1}\left(A \backslash\left\{a_{1}, a_{2}\right\}\right)$ and $T=\bar{f}^{-1}\left(a_{1}\right)$. There is a homeomorphism $h: M \rightarrow M$ generating the $Z_{k}$ action such that the pair $(U, T)$ forms an $h$-partition [5] with both $U$ and $T$ connected. This means that $M=\bigcup_{i=1}^{k}\left[h^{i}(U) \cup h^{i}(T)\right], \operatorname{Bd}(U)=T \cup h(T)$, and $h^{i}(T)$ $\cap h^{j}(T)=\varnothing$ and $h^{i}(U) \cap h^{j}(U)=\varnothing$ if $i \neq j(\bmod k)$.

By using the fact that $h \simeq 1_{M}$ we show in [5] that $\pi_{1}(M)$ is an extension of $\pi_{1}(T)$ by the infinite cyclic group $Z$. Now split $M$ along $T$ to obtain a manifold $E$ with boundary two disjoint copies $T_{0}, T_{1}$ of $T$. The maps $\pi_{1}\left(T_{0}\right) \rightarrow \pi_{1}(E)$ and $\pi_{1}\left(T_{1}\right) \rightarrow \pi_{1}(E)$ are isomorphisms. Stallings shows in the proof of Theorem 2 of $[4]$ that $E \approx T \times[0,1]$. The homeomorphism $\psi: T \rightarrow T$ repairing this cut (i.e. $M \approx T \times$ $R /\{(x, t) \sim(\psi(x), t+1)\}=T \times R / \psi)$ can be chosen so that $\psi$ is of finite order $n$, where $(n, k)=1$ [6]. For convenience in notation, we identify $M$ with $T \times R / \psi$, and denote points in $M$ by $[x, t]$.

In $E$ we have $h^{i}(T)$ separating $T_{0}$ from $T_{1}$ for $1 \leqq i<k$. It follows from Theorem 7.2 of [1] that $h: M \rightarrow M$ is equivalent to a homeomorphism, again denoted by $h$, such that in $E$ we have $h^{i}(T)=T$ $\times\{i / k\}(1 \leqq i \leqq k)$. The new $h$ may clearly be chosen so that in $E$ we have $h^{i}: T \times\{\lambda\} \rightarrow T \times\{\lambda+i / k\}$ for all $\lambda \geqq 0$ with $\lambda+i / k \leqq 1$ $(i=1, \cdots, k-1)$.

Let $\rho: T \times[0,1] \rightarrow T$ be the projection to the first factor. Since $h^{k}=1_{M}$, we may conclude that the composite

$$
T=T \times\{0\} \stackrel{h^{k}}{\rightarrow} T \times\{1\} \subset T \times[0,1] \stackrel{\rho}{\rightarrow} T
$$

is equivalent to $\psi: T \rightarrow T$, the homeomorphism repairing the cut of $M$ along $T$.

Let $h_{1}: T \rightarrow T$ be the homeomorphism defined by the composite 


$$
T=T \times\{0\} \stackrel{h}{\rightarrow} T \times\{i / k\} \subset T \times[0,1] \stackrel{\rho}{\rightarrow} T .
$$

Then $\left(h_{1}\right)^{k}=\psi$ and $h[x, t]=\left[h_{1}(x), t+1 / k\right]$. Let $g: M \rightarrow M$ be any homeomorphism such that $g[x, t]=\left[g_{1}(x), t\right]$. Consider the infinite covering of $M=T \times R / \psi$ by $T \times R$. Observe that $h$ is homotopic to $g$ if and only if $h_{1}^{-1} g_{1}$ is homotopic (and hence isotopic) to some power of $\psi=\left(h_{1}\right)^{k}$. By hypothesis $h \simeq 1_{M}$. Hence some power of $\left(h_{1}\right)^{k}$ is isotopic to $h_{1}$. It follows that the order of $h_{1}$ is relatively prime with respect to $k$ and is equal to $n$, the order of $\psi$.

To define an $S O(2)$ action on $M$, we identify the circle with $R / Z$ and denote elements as $[s]$, for $s \in R$. We define the continuous map $S^{1} \times M \rightarrow M$ by $[s] \times[x, t] \mapsto[x, n s+t]$. It is easy to check that this is a well-defined effective action of $S O(2)$ without fixed points. The free $Z_{k}$ action imbedded in this $S O(2)$ action is generated by the homeomorphism $\gamma:[x, t] \mapsto[x, t+1 / k]$. We have $a n+b k=1$ for some integers $a, b$. A computation shows that $\gamma^{a n}[x, t]=\left[\psi^{b}(x), t+1 / k\right]=$ $\left[h_{1}(x), t+1 / k\right]$. Therefore $\gamma^{a n}=h$.

CoRollary 1. If a closed, orientable, irreducible 3-manifold $M$ admits a proper free $Z_{k}$ action ( $k \geqq 2$ and prime) that is $Z$-classified, then the orbit space $M^{*}$ fibers over the circle and admits an effective $S O(2)$ action without fixed points.

Corollary 2. Suppose a closed, orientable, irreducible 3-manifold $M$ admits a $Z$-classified, proper, free $Z_{k}$ action ( $k \geqq 2$ and prime). Then there is only one such $Z_{k}$ action on $M$ (up to weak equivalence).

Proof. This follows from the uniqueness of the effective $S O(2)$ action on $M$ in which such a $Z_{k}$ action is imbedded [3, Theorem 4]. $\square$

\section{REFERENCES}

1. E. M. Brown, Unknotting in $M^{2} \times I$, Trans. Amer. Math. Soc. 123 (1966), 480505. MR 33 \#6640.

2. P. Orlik, Examples of free involutions on 3-manifolds, Proc. Conference Transformation Groups, Springer-Verlag, Berlin, 1968, pp. 205-206.

3. P. Orlik and R. Raymond, Actions of SO(2) on 3-manifolds, Proc. Conference Transformation Groups, Springer-Verlag, Berlin, 1968, pp. 297-318.

4. J. Stallings, On fibering certain 3-manifolds, Topology of 3-Manifolds and Related Topics (Proc. Univ. of Georgia Inst. 1961) Prentice-Hall, Engelwood Cliffs, N.J., 1962, pp. 95-100. MR $28 \# 1600$.

5. J. L. Tollefson, Fibering 3-manifolds that admit free $Z_{k}$ actions, Trans. Amer. Math. Soc. 147 (1970), 279-287.

6. - A characterization of 3-manifolds that are products, Amer. J. Math. 90 (1970).

7. F. Waldhausen, Gruppen mit Zentrum und 3-dimensionale Mannigfaltigkeiten, Topology 6 (1967), 505-517. MR 38 \#5223.

Tulane University, New Orleans, Louisiana 70118 